\title{
Usefulness of apical area index to predict left ventricular thrombus in patients with systolic dysfunction: a novel index from cardiac magnetic resonance
}

\author{
Yodying Kaolawanich and Thananya Boonyasirinant ${ }^{*}$ (i)
}

\begin{abstract}
Background: LV systolic dysfunction presents an elevated risk of thromboembolism. Previous studies demonstrated low left ventricular ejection fraction (LVEF), ischemic cardiomyopathy and increased myocardial scarring as independent risk factors for LV thrombus formation. Structural changes that alter the size and shape of LV apex may have a significant role in predicting LV thrombus, but there is no definite evidence exists in this entity.

Methods: A case-control cardiac magnetic resonance (CMR) study of 150 patients with LV systolic dysfunction (LVEF < 40\%; 30 patients with LV thrombus and 120 patients without thrombus) was performed. Factors associated with thrombus including sphericity index and 'new' apical area index (ratio of apical area to entire LV area from a cine four-chamber view) were evaluated.

Results: Average age was $63.48 \pm 12.82$ years and mean LVEF was $29.22 \pm 8.53 \%$. Patients with LV thrombus had significantly higher apical area index than those without thrombus ( $46.5 \pm 3.27$ vs. $42.71 \pm 3.02, p<0.001)$ while sphericity index in both groups was not different (1.63 \pm 0.27 vs. $1.67 \pm 0.19, p=0.57)$. Univariate analysis revealed that male gender, prior myocardial infarction, presence of apical aneurysm, ischemic-typed scar, apical scar and apical area index were associated with thrombus. Further, multivariate analysis showed only apical area index and apical scar as independent predictors for thrombus formation.
\end{abstract}

Conclusion: Apical area index from CMR is a new index to predict LV thrombus in patients with LV systolic dysfunction and may have a future role in early anticoagulant therapy.

Keywords: Thrombus, Systolic dysfunction, Left ventricular geometry, Cardiac magnetic resonance, Apical area index

\section{Introduction}

Patients with left ventricular (LV) systolic dysfunction are at increased risk of thromboembolic events that may result in major adverse cardiac sequelae. According to previous echocardiographic studies, the prevalence of LV thrombus in patients with LV systolic dysfunction was $8-19 \%[1,2]$. Thus, accurate detection of LV thrombus affects clinical outcomes and appropriate management.

* Correspondence: drthananyaa@yahoo.com

Division of Cardiology, Department of Medicine, Siriraj Hospital, Mahidol

University, 2 Wanglang Road, Bangkoknoi, Bangkok 1070, Thailand
Cardiac magnetic resonance (CMR) provides highresolution images, good reproducibility and tissue characterization for detection of thrombus [3]. Various CMR sequences are dedicated for thrombus identification with excellent diagnostic accuracy, and sensitivity and specificity at 88 and $99 \%$, respectively [4]. These include gradient-echo, steady-state free precession (SSFP), and late gadolinium enhancement (LGE).

A recent comparative study demonstrated that CMR yielded more than 3-fold higher diagnostic accuracy for detection of LV thrombus compared with transthoracic and transesophageal echocardiography which detected

(c) The Author(s). 2019 Open Access This article is distributed under the terms of the Creative Commons Attribution 4.0 International License (http://creativecommons.org/licenses/by/4.0/), which permits unrestricted use, distribution, and reproduction in any medium, provided you give appropriate credit to the original author(s) and the source, provide a link to the Creative Commons license, and indicate if changes were made. The Creative Commons Public Domain Dedication waiver (http://creativecommons.org/publicdomain/zero/1.0/) applies to the data made available in this article, unless otherwise stated. 
thrombus based on anatomical appearance rather than tissue characteristics [4].

Two CMR studies demonstrated independent predictors of LV thrombus such as worsening left ventricular ejection fraction (LVEF), ischemic etiology, prior anterior wall myocardial infarction, and increased myocardial scarring [5, 6]. Apart from these factors, LV shape and geometrical changes may be associated with thrombus formation. However, no definite geometrical index exists to accurately predict thrombus.

Previous echocardiographic studies reported that some parameters including sphericity index (long-to-short axis ratio in a four-chamber view) and apical conicity index (apical-to-short axis length ratio in a four-chamber view) affected LV remodeling after anterior wall myocardial infarction [7]. Nevertheless, these indexes were not studied for thrombus prediction.

From our hypothesis, apical remodeling may associate with thrombus formation. Previous indices involved particular length measurements without a comprehensive evaluation of LV apex, thus we would like to introduce 'apical area index' (ratio of apical area to entire LV in a cine four-chamber view) as a new predictor of LV thrombus. The primary objective of this study was to compare apical area index of patients with LV thrombus with no thrombus group. The secondary objective was to determine the predictors of LV thrombus in patients with systolic dysfunction.

\section{Methods}

\section{Study population}

This case-control, single center study was approved by the institutional ethics committee. A total of 150 patients with systolic dysfunction (30 consecutive cases with thrombus and 120 consecutive controls without thrombus) who underwent cine, adenosine stress, and LGE-CMR during the same period at Siriraj Hospital (Mahidol University, Bangkok, Thailand) between August 2014 and January 2016 were enrolled. Systolic dysfunction was defined as LVEF by cine-CMR below $40 \%$. Patients were most commonly referred for CMR examination to assess myocardial ischemia, myocardial viability, or causes of cardiomyopathy. Patients exhibiting the following criteria were excluded: 1 ) inability to perform CMR due to a permanent pacemaker or implantable cardioverter defibrillator implantation, 2) incomplete CMR examination, 3) history of allergy to gadolinium, 4) pregnancy, and (5) history of claustrophobia.

On the day of the CMR procedure, a complete medical history including cardiac risk factors, current medications including antithrombotic and heart failure drugs, information regarding coronary angiography results, prior coronary revascularization, previous myocardial infarction, and thromboembolic events were collected to assess potential predictors of thrombus.

Additionally, regarding the excellent ability of LGE-CMR for the diagnosis of infarcted myocardium, patients were considered to have ischemic cardiomyopathy if there was transmural or subendocardial scar pattern from LGE-CMR. All other patients were classified as having nonischemic cardiomyopathy.

\section{CMR protocol}

Cardiac structure, function and thrombus were assessed from two CMR techniques, cardiac function by a SSFP technique and LGE using a 1.5 Tesla Gyroscan NT Philips scanner (Philip Medical Systems, Best, The Netherlands). Functional study was performed by the acquisition of images by a SSFP technique in Short-axis images were acquired every $8 \mathrm{~mm}$ throughout the entire LV. Longaxis images were obtained in standard 2-, 3-, and fourchamber orientations. Parameters for cardiac function were as follows: repetition time/echo time/number of excitations $=3.7 \mathrm{~ms} / 1.8 \mathrm{~ms} / 2,390 \times 312 \mathrm{~mm}$ field of view, $256 \times 240$ matrix, $1.52 \times 1.21$ reconstruction pixel, $8 \mathrm{~mm}$ slice thickness, and 70 flip angles.

For LGE imaging, images were acquired 15 to $20 \mathrm{~min}$ after intravenous injection of $0.2 \mathrm{mmol} / \mathrm{kg}$ gadolinium contrast agent (Magnevist, Bayer Schering Pharma, Berlin, Germany) with the following scanning parameters; echo time $1.25 \mathrm{~ms}$, repetition time $4.1 \mathrm{~ms}$, 15-degree flip angle, $303 \times 384 \mathrm{~mm}$ field of view, $240 \times 256$ matrix, in-plane resolution $1.26 \times 1.5 \mathrm{~mm}$, slice thickness $8 \mathrm{~mm}$ and 1.5 Sensitivity-Encoding (SENSE) factor.

Additionally, long inversion time sequence was applied for increasing the accuracy for detection of LV thrombus, as mentioned in previous study [5].

\section{CMR analysis}

CMR indices of LV function, geometry, and scarring were measured to determine whether these parameters were related to the presence of thrombus. CMR images in short-axis view were classified as the basal, mid, or apical part of the left ventricle. Segmentation of each slice was performed according to the recommendation of American Heart Association [8]. Left ventricular volume and mass was calculated and indexed for the body surface area. LVEF was quantitatively assessed by using end-systolic and end-diastolic volume calculated from the multiple slice short axis images. Wall motion of each myocardial segment was recorded as presence or absence of abnormal wall motion. Wall motion of each myocardial segment was also recorded as 5 -grade system as follows: 1 =normal, 2 =hypokinesia, 3 = akinesia, $4=$ dyskinesia, and $5=$ aneurysm. LGE images were analyzed by visual assessment. The transmural extent of LGE was graded as follows: $0=$ no LGE, $1=1-25 \%, 2=26-50 \%$, 
$3=51-75 \%$, and $4=76-100 \%$ compared to the myocardial area in that segment. Total scar volume as a percentage of LV myocardium was defined as the sum of all transmural extent of infarction scores throughout LV wall thickness / 4 times total number of segments [9]. Transmural scar was defined as transmural extension of LGE more than $50 \%$ of myocardial thickness in each segment, subendocardial scar was defined as transmural extension of LGE less than $51 \%$ of myocardial thickness.

\section{LV thrombus assessment}

Left ventricular thrombi detected by CMR on cine sequences were defined as filling defects within the LV cavity, typically adherent to regions of abnormal wall motion (hypokinesis, akinesis, or dyskinesis) then confirmed by LGE technique in Fig. 1a. In LGE images, thrombus was diagnosed as an LV mass with post-contrast inversion-recovery characteristics consistent with avascular tissue. Moreover, LGE-CMR can be further assessed for thrombus identification by increasing the inversion time (i.e., $600 \mathrm{msec}$ ) to selectively null avascular tissue such as thrombus. This 'long inversion time' approach provides an image that renders black thrombus and surrounding bright myocardium (Fig. 1b) [10].

\section{Geometrical parameters calculation}

Here, two indices were evaluated as sphericity index and 'new' apical area index. The sphericity index was described as the LV long-axis length divided by the mid LV diameter at end diastole for each in Fig. 2 [11, 12].

To represent the importance of apical remodeling, a 'new' apical area index was introduced to evaluate the predictor of thrombus. The apical area index was simply calculated as a percentage of an apical-half LV area (mid to apex) divided by an entire LV area from a cine four-chamber view in end diastole. Similar to the sphericity index, LV long-axis length was measured from the endocardial border of the mitral valve annulus plane to tip of apex, and the mid LV diameter was measured at the half-length from the LV long axis to tip of apex (Fig. 3).

\section{Intra- and inter-observer reliability}

To assess intra- and inter-observer reliability of apical area index measurements by CMR, 20 patients were randomly selected to measure variability by the same observer 4 weeks after the initial analysis, and by the second independent observer blinded to the initial results.

\section{Statistical methods}

Descriptive statistics, including frequency and percentage, were used for categorical variables. Continuous variables were reported as mean \pm standard deviation for normally distributed variables and median (percentile 25 and percentile 75) for non-normally distributed variables. Normality of distribution of variables was examined by Kolmogorov-Smirnov test. Comparisons of categorical variables between patients with and without thrombus were performed using chi-square test or Fisher's exact test. Continuous variables were compared using Student's t-test or Mann-Whiney U test. Univariate and Multivariate analyses of thrombus predictions were evaluated using binary logistic regression analysis (Backward method) and presented as Odds ratio (OR) (95\% confidence interval [CI]). Intraand inter-observer reliability were analyzed by the intraclass correlation coefficient.

For all tests performed, a two-tailed $p$-value $<0.05$ was statistically significant. PASW Statistic (SPSS) 18.0 (SPSS, Inc., Chicago, IL, USA) was used to perform all statistical analyses.
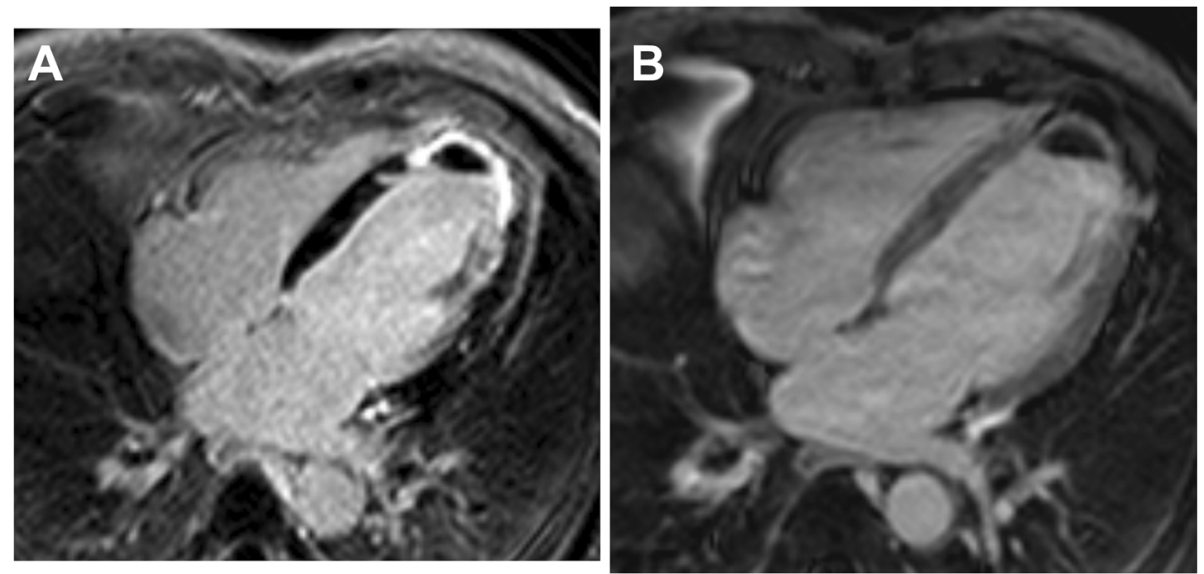

Fig. 1 Presence of LV thrombus from LGE study. a LV thrombus from standard LGE. b LV thrombus from long inversion time sequence 


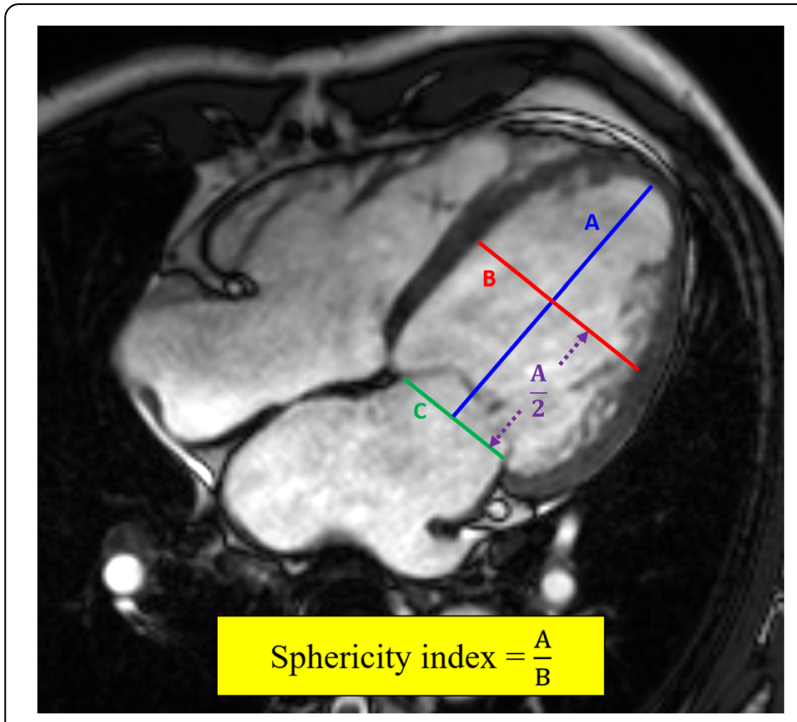

Fig. 2 Calculation of sphericity index

\section{Results}

\section{Population characteristics}

Clinical characteristics of the overall population are shown in Table 1. In total, 150 patients (103 male and 47 female) were consecutively enrolled with mean age of $63.84 \pm 12.82$ years. Almost a quarter of the subjects had prior myocardial infarction. Sixteen patients had history of a prior cerebrovascular event and $15.3 \%$ were receiving chronic warfarin therapy.

Imaging parameters are demonstrated in Table 2. The cine-CMR showed evidence of advanced systolic dysfunction. Average LVEF was $29.22 \pm 8.53 \%$ with apical left ventricular aneurysms presented in $22 \%$. Extensive scarring was demonstrated from LGE images with mean scar volume $29.76 \pm 15.72 \%$. Transmural and subendocardial scar in the coronary vascular territory was defined as ischemic scarring pattern and found in 91 patients (60.7\%). Apical scar was present in 47 patients $(31.3 \%)$.

\section{Clinical and structural markers of the presence of thrombus}

Thirty patients with LV thrombus from cine and LGECMR were mostly male and exhibited prior myocardial infarction without any differences in other coronary artery disease risk factors, atrial fibrillation, prior thromboembolic events, or baseline medication regimen including anticoagulation therapy.

Patients with LV thrombus did not differ from those without thrombus in aspects of LV volume and LVEF. Apical aneurysm was presented in significantly more patients with LV thrombus than without $(50 \%$ vs. $15.8 \%, p$-value $<0.0001)$. Significantly extensive apical scarring was found in patients with LV thrombus compared with no thrombus $(63.3 \%$ vs $23.3 \%$, $p$-value $<0.0001)$.

For LV geometrical indexes, patients with LV thrombus had significantly greater apical area index than those without thrombus $(46.5 \pm 3.27$ vs $42.71 \pm 3.02$, $p$-value $<0.0001$ ), while no difference of sphericity index was detected between patients with and without thrombus $(1.63 \pm 0.27$ vs $1.67 \pm 0.19$, p-value $=0.57)$.

\section{Multivariate analysis}

Six clinical and CMR variables including male gender, history of myocardial infarction, presence of apical aneurysm, ischemic cardiomyopathy scar pattern, apical scar, and apical area index were analyzed using multivariate analysis (Table 3). Only the presence of apical scar and apical area index were determined as independent markers for thrombus.

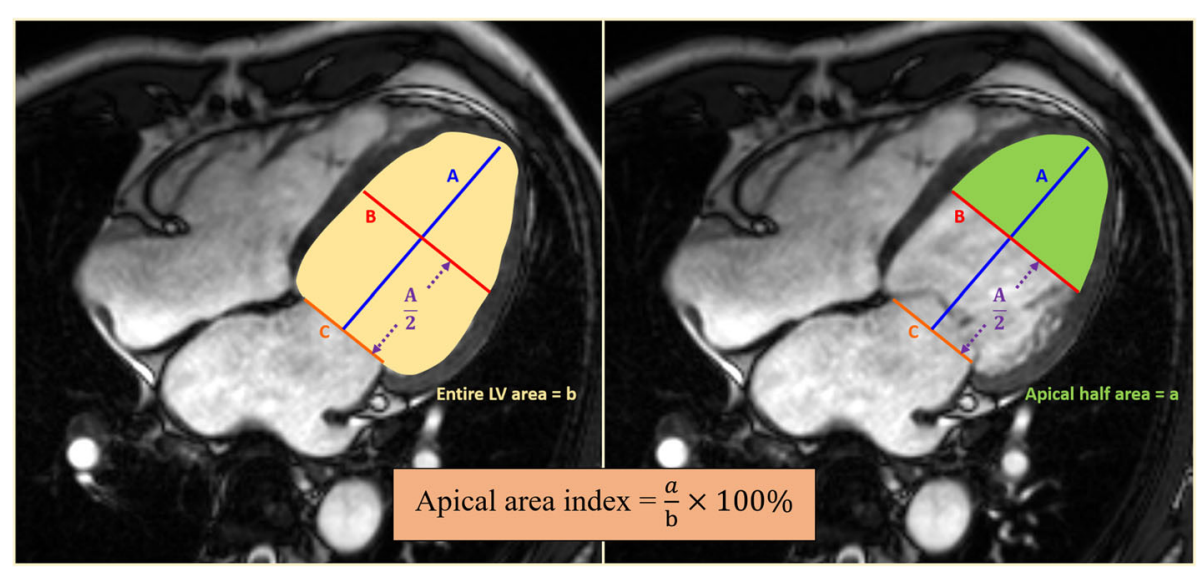

Fig. 3 Calculation of apical area index 
Table 1 Baseline characteristics

\begin{tabular}{|c|c|c|c|c|}
\hline & Total $(n=150)$ & Thrombus $(n=30)$ & No thrombus $(n=120)$ & $p$-value \\
\hline Age (years) & $63.84 \pm 12.82$ & $60.93 \pm 14.20$ & $64.57 \pm 12.41$ & 0.17 \\
\hline Male gender & $103(68.7 \%)$ & $26(86.7 \%)$ & 77 (64.2\%) & 0.02 \\
\hline BMI (kg/sqm) & $24.27 \pm 4.42$ & $25.66 \pm 5.50$ & $23.92 \pm 4.06$ & 0.06 \\
\hline \multicolumn{5}{|l|}{ Atherosclerotic risk factors } \\
\hline Hypertension & $110(73.3 \%)$ & $26(86.7 \%)$ & $84(70 \%)$ & 0.07 \\
\hline Diabetes mellitus & 49 (32.7\%) & $12(40 \%)$ & $37(30.8 \%)$ & 0.34 \\
\hline Dyslipidemia & $108(72 \%)$ & $26(86.7 \%)$ & $82(68.3 \%)$ & 0.05 \\
\hline Smoking & $22(14.7 \%)$ & $4(13.3 \%)$ & $18(15 \%)$ & 1.00 \\
\hline History of myocardial infarction & $36(24 \%)$ & $13(43.3 \%)$ & $23(19.2 \%)$ & 0.006 \\
\hline Coronary revascularization (PCI) & $9(6 \%)$ & $2(6.7 \%)$ & $7(5.8 \%)$ & 1.00 \\
\hline History of heart failure & $88(58.7 \%)$ & $19(63.3 \%)$ & $69(57.5 \%)$ & 0.56 \\
\hline History of CVA or TIA & $16(10.7 \%)$ & $4(13.3 \%)$ & $12(10 \%)$ & 0.53 \\
\hline Atrial fibrillation or atrial flutter & $26(17.3 \%)$ & $3(10 \%)$ & $23(19.2 \%)$ & 0.24 \\
\hline \multicolumn{5}{|l|}{ Antithrombotic } \\
\hline Aspirin & 96 (64\%) & $21(70 \%)$ & 75 (62.5\%) & 0.44 \\
\hline Warfarin & $23(15.3 \%)$ & $2(6.7 \%)$ & $21(17.5 \%)$ & 0.17 \\
\hline \multicolumn{5}{|l|}{ Heart failure medications } \\
\hline Beta-blocker & $113(75.3 \%)$ & $22(73.3 \%)$ & $91(75.8 \%)$ & 0.78 \\
\hline ACEI & $64(42.7 \%)$ & $14(46.7 \%)$ & $50(41.7 \%)$ & 0.62 \\
\hline Angiotensin receptor blocker & $32(21.3 \%)$ & $4(13.3 \%)$ & $28(23.3 \%)$ & 0.23 \\
\hline Spironolactone & $23(15.3 \%)$ & $2(6.7 \%)$ & $21(17.5 \%)$ & 0.17 \\
\hline Furosemide & $72(48 \%)$ & $13(43.3 \%)$ & $59(49.2 \%)$ & 0.57 \\
\hline Nitrate & $40(26.7 \%)$ & $9(30 \%)$ & $31(25.8 \%)$ & 0.64 \\
\hline Digoxin & $20(13.3 \%)$ & $3(10 \%)$ & $17(14.2 \%)$ & 0.77 \\
\hline
\end{tabular}

$A C E I=$ angiotensin converting enzyme inhibitors, $B M I=$ body mass index,$C V A=$ cerebrovascular accident,$P C l=$ percutaneous coronary intervention, $T I A=$ transient ischemic attack

Table 2 CMR parameters

\begin{tabular}{|c|c|c|c|c|}
\hline & $\begin{array}{l}\text { Total } \\
(n=150)\end{array}$ & $\begin{array}{l}\text { Thrombus } \\
(n=30)\end{array}$ & $\begin{array}{l}\text { No thrombus } \\
(n=120)\end{array}$ & $p$-value \\
\hline \multicolumn{5}{|l|}{ LV function and morphology } \\
\hline End-diastolic volume (ml) & $238.87 \pm 60.02$ & $242.47 \pm 55.91$ & $236.71 \pm 61.05$ & 0.38 \\
\hline End-systolic volume (ml) & $172.07 \pm 57.75$ & $181.90 \pm 62.38$ & $169.61 \pm 56.54$ & 0.30 \\
\hline Left ventricular ejection fraction (\%) & $29.22 \pm 8.53$ & $28.20 \pm 10.38$ & $29.48 \pm 8.03$ & 0.47 \\
\hline Left ventricular mass index (g/sqm) & $76.16 \pm 22.60$ & $79.41 \pm 22.58$ & $75.35 \pm 22.63$ & 0.38 \\
\hline Presence of apical aneurysm & $34(22.7 \%)$ & $15(50 \%)$ & 19 (15.8\%) & $<0.0001$ \\
\hline \multicolumn{5}{|l|}{ LV scarring } \\
\hline Ischemic cardiomyopathy scar & $91(60.7 \%)$ & $25(83.3 \%)$ & $66(55 \%)$ & 0.004 \\
\hline Scar volume (\% LV) & $29.76 \pm 15.72$ & $34.06 \pm 14.69$ & $27.97 \pm 15.91$ & 0.06 \\
\hline Presence of apical scar & $47(31.3 \%)$ & $19(63.3 \%)$ & $28(23.3 \%)$ & $<0.0001$ \\
\hline \multicolumn{5}{|l|}{ Shape parameters } \\
\hline Sphericity index & $1.64 \pm 0.21$ & $1.63 \pm 0.27$ & $1.67 \pm 0.19$ & 0.57 \\
\hline Apical area index & $43.46 \pm 3.42$ & $46.50 \pm 3.27$ & $42.71 \pm 3.02$ & $<0.0001$ \\
\hline
\end{tabular}


Table 3 Univariate and multivariate analysis

\begin{tabular}{|c|c|c|c|c|}
\hline & \multicolumn{2}{|l|}{ Univariate analysis } & \multicolumn{2}{|c|}{ Multivariate analysis } \\
\hline & OR $(95 \% \mathrm{Cl})$ & $p$-value & OR $(95 \% \mathrm{Cl})$ & $p$-value \\
\hline Male gender & $3.63(1.19-11.09)$ & 0.02 & - & - \\
\hline History of myocardial infarction & $3.23(1.37-7.57)$ & 0.007 & - & - \\
\hline Presence of apical aneurysm & $5.32(2.23-12.66)$ & $<0.0001$ & - & - \\
\hline Ischemic cardiomyopathy scar & $4.09(1.47-11.40)$ & 0.007 & - & - \\
\hline Presence of apical scar & $5.68(2.42-13.34)$ & $<0.0001$ & $3.56(1.07-11.86)$ & 0.04 \\
\hline Apical area index & $1.49(1.27-1.75)$ & $<0.0001$ & $1.51(1.23-1.84)$ & $<0.0001$ \\
\hline
\end{tabular}

\section{Intra- and inter-observer reliability}

Moderate intra- and inter-observer reliability were shown for apical area index measurements by cine-CMR. In 20 patients, mean apical area index values were $44.05 \pm 4.79$ and $43.18 \pm 4.13(r=0.73, p=0.001)$ for the first observer in the initial analysis and 4 weeks later, respectively, and $42.52 \pm 3.51(r=0.69, p=0.001)$ for the second observer in the initial analysis.

\section{Discussion}

Recent studies have shown independent risk factors for LV thrombus formation including low LVEF, ischemic cardiomyopathy, prior anterior wall myocardial infarction, and increased myocardial scarring $[5,6]$. However, an established geometrical parameter for prediction of LV thrombus has remained unclear. Our study provides a new index for thrombus formation as 'apical area index'. Patients with thrombus demonstrated significantly greater apical area index than without thrombus. Furthermore, our study demonstrated apical scarring as another strong independent predictor of LV thrombus.

\section{CMR evaluation of LV thrombus}

Accurate detection of cardiac thrombus affects clinical outcomes and therapeutic management as LV thrombus provides a substrate for thromboembolic events and a rationale for anticoagulation. CMR enables LV thrombus to be detected based on intrinsic tissue characteristics related to avascular tissue composition. CMR tissue characterization for LV thrombus has been well-documented and validated compared to both pathological and clinical outcome reference standards.

LGE-CMR is widely used to differentiate between infarcted and viable myocardium based on relative differences in gadolinium-based contrast uptake. The technique can be used to identify thrombus and has been well validated in several different at-risk cohorts.

Long inversion time is another CMR sequence add-on standard LGE-CMR which increases the accuracy of thrombus detection, especially for mural thrombi which are difficult to identify by standard LGE imaging. Prolonging the inversion time produces an image that renders thrombus black with surrounding bright myocardium and accentuates the utility of CMR to evaluate patients with LV systolic dysfunction, as in our study.

Recent comparative studies have demonstrated that CMR yields superior detection of LV thrombus compared with echocardiography, which detects thrombus based on anatomical appearance rather than tissue characteristics. Among 160 patients undergoing LV reconstruction surgery (in whom pathology verification was uniformly available), Srichai et al. reported that CMR yielded more than 3-fold higher diagnostic accuracy for detection of thrombus then transthoracic echocardiography ( $87 \%$ vs. $27 \%)$ [4]. Improved accuracy was predominantly attributable to markedly higher sensitivity for CMR compared to echocardiography (88\% vs. 23\%).

Screening algorithms have been proposed that use the extent of wall-motion abnormalities on transthoracic echocardiography to trigger consideration of subsequent imaging with CMR [13]. High apical LV wall motion scores yield sensitivity approaching $100 \%$, with specificity greater than $60 \%$, and low apical LV wall motion scores allow appropriate avoidance of further testing in a substantial proportion of patients.

Previous evidence indicated CMR as a non-invasive modality of choice to diagnose LV thrombus. Moreover, our study emphasized the importance of apical remodeling to provide apical area index assessment.

\section{Markers of thrombus formation}

From our study, patients with LV thrombus did not differ from those without thrombus in aspects of LV volume and systolic function, while male gender, history of myocardial infarction, apical aneurysm, apical scar, and ischemic scar were higher in the thrombus group. Most factors corresponded with prior studies such as history of myocardial infarction, ischemic scar and apical aneurysm [5, 14-17]. Apical aneurysm was found in 50\% of patients with LV thrombus and was an important predictor of severe apical asynergy in our study.

We determined apical scarring as a new predictor of LV thrombus. Extensive apical scarring was found significantly more in patients with thrombus than without 
thrombus $(63.3 \%$ vs $23.3 \%, p$-value $<0.0001)$ as additional evidence demonstrating the significance value of apical change for thrombus formation.

Scar volume has previously been established as a risk factor for thrombus formation [5]. Our study found no difference of scar volume between patients with LV thrombus and those without. However, patients in both groups showed evidence of severe scarring (mean scar volume of all patients $=29.76 \pm 15.72 \%$ LV) which was higher than previous studies. A trend of greater scar volume was presented in the thrombus group $34.06 \%$ vs $27.97 \%, p=0.06$ ).

\section{New apical index to fill the gap}

Sphericity index and apical area index were the two geometrical parameters assessed for LV thrombus prediction in our study. The sphericity index was described according to substantial echocardiographic studies which evaluated LV systolic and diastolic dysfunction [11, 12]. One CMR study indicated that increased sphericity index was associated with reduced apical relaxation velocities as previously described [18]. Our hypothesis suggested that apical shape may be associated with LV thrombus formation. The sphericity index originates from the ratio of LV long axis to short axis in four-chamber view that may represent apical remodeling, while apical area index provides more information as it calculates the apex area ratio to entire LV, not only from dimensional evaluation.

From our results, sphericity index failed to demonstrate any difference between the two groups, while apical area index in the LV thrombus group was significantly higher than the group without thrombus. Apical area index measurement is simple with acceptable reproducibility and add-on information to predict LV thrombus in patients with systolic dysfunction.

Moreover, from multivariate analysis, only apical scar and apical area index were determined as independent predictors of LV thrombus. Combinations of these two factors will provide additional value in this aspect. This new index in other cardiomyopathies involving apex warrants further study.

\section{Study limitations}

There were some study limitations, First, our study design cannot demonstrate prevalence of LV thrombus in the population; however, this was evident in previous study [5]. Second, a case-control study can only show an association between a predictor and an outcome without guaranteeing a causal relationship. Third, too few patients had undergone coronary angiography to provide a definite diagnosis of ischemic cardiomyopathy. Nevertheless, this study focused mainly on CMR parameters and the accuracy of LGE technique to differentiate these conditions was excellent. Forth, our study was a small single center study to initially prove the hypothesis. Further study which includes more patients or multicenter study is necessary for the validation of the results.

\section{Conclusion}

Apical area index is a new simple predictor of $\mathrm{LV}$ thrombus formation from cine-CMR in patients with systolic dysfunction.

\section{Clinical application and future research}

Results will be of value for two future research areas as 1) to identify the cut-off level of apical area index to accurately predicted thrombus in larger populations, and 2) to evaluate the association between apical area index and hard clinical outcomes such as stroke. If an association is present, patients may get benefits from early coagulation therapy. This entity warrants further study.

\section{Abbreviations}

ACEl: Angiotensin converting enzyme inhibitors; BMl: Body mass index; CMR: Cardiac magnetic resonance; CVA: Cerebrovascular accident; LGE: Late gadolinium enhancement; LV: Left ventricular / ventricle; LVEF: Left ventricular ejection fraction; $\mathrm{PCl}$ : Percutaneous coronary intervention; SSFP: Steady state free precession; TIA: Transient ischemic attack

\section{Acknowledgements}

Miss Khemajira Karaketklang, M.P.H. (Biostatistics).

Funding

None.

Authors' contributions

Both authors read and approved the final manuscript.

Competing interests

The authors declare that they have no competing interests.

\section{Publisher's Note}

Springer Nature remains neutral with regard to jurisdictional claims in published maps and institutional affiliations.

Received: 24 October 2018 Accepted: 20 December 2018

Published online: 11 January 2019

\section{References}

1. Gottdiener JS, Massie B, Ammons SB, Egher C, Petillo F, Krol WF, et al. Prevalence of left ventricular thrombus in dilated cardiomyopathy: The WATCH trial. J Am Coll Cardiol. 2003:41(6):202a.

2. Sharma ND, McCullough PA, Philbin EF, Weaver WD. Left ventricular thrombus and subsequent thromboembolism in patients with severe systolic dysfunction. Chest. 2000;117(2):314-20.

3. Grothues F, Smith GC, Moon JCC, Bellenger NG, Collins P, Klein HU, et al. Comparison of interstudy reproducibility of cardiovascular magnetic resonance with two-dimensional echocardiography in normal subjects and in patients with heart failure or left ventricular hypertrophy. Am J Cardiol. 2002;90(1):29-34.

4. Srichai $M B$, Junor $C$, Rodriguez $L L$, Stillman $A E$, Grimm RA, Lieber $M L$, et al. Clinical, imaging, and pathological characteristics of left ventricular thrombus: a comparison of contrast-enhanced magnetic resonance imaging, transthoracic echocardiography, and transesophageal echocardiography with surgical or pathological validation. Am Heart J. 2006; 152(1):75-84.

5. Weinsaft JW, Kim HW, Shah DJ, Klem I, Crowley AL, Brosnan R, et al. Detection of left ventricular thrombus by delayed-enhancement 
cardiovascular magnetic resonance - prevalence and markers in patients with systolic dysfunction. J Am Coll Cardiol. 2008;52(2):148-57.

6. Cambronero-Cortinas E, Bonanad C, Monmeneu JV, Lopez-Lereu MP, Gavara $J$, de Dios E, et al. Incidence, outcomes, and predictors of ventricular Thrombus after Reperfused ST-segment-elevation myocardial infarction by using sequential cardiac MR imaging. Radiology. 2017;284(2):372-80.

7. Di Donato M, Dabic P, Castelvecchio S, Santambrogio C, Brankovic J, Collarini $\mathrm{L}$, et al. Left ventricular geometry in normal and post-anterior myocardial infarction patients: sphericity index and 'new' conicity index comparisons. Eur J Cardio-Thorac. 2006;29:S225-S30.

8. Cerqueira MD, Weissman NJ, Dilsizian V, Jacobs AK, Kaul S, Laskey WK, et al. Standardized myocardial segmentation and nomenclature for tomographic imaging of the heart - a statement for healthcare professionals from the cardiac imaging Committee of the Council on clinical cardiology of the American Heart Association. Circulation. 2002;105(4):539-42.

9. Wagner A, Mahrholdt H, Holly TA, Elliott MD, Regenfus M, Parker M, et al. Contrast-enhanced MRI and routine single photon emission computed tomography (SPECT) perfusion imaging for detection of subendocardial myocardial infarcts: an imaging study. Lancet. 2003;361(9355):374-9.

10. Shah DJ, Judd RM, Kim RJ. Myocardial viability. In: Edelman RR, Hesselink JR, Zlatkin MB, et al., editors. Clinical Magnetic Resonance Imaging. 3rd ed. New York, NY: Elsevier; 2006

11. Tischler MD, Ashikaga T, LeWinter MM. Relation between left ventricular shape and Doppler filling parameters in patients with left ventricular dysfunction secondary to coronary artery disease. Am J Cardiol. 1995;76: 553-6.

12. Tischler MD, Niggel J, Borowski DT, LeWinter MM. Relation between left ventricular shape and exercise capacity in patients with left ventricular dysfunction. J Am Coll Cardiol. 1993;22:751-7.

13. Weinsaft JW, Kim J, Medicherla CB, et al. Echocardiographic algorithm for post-myocardial infarction Iv thrombus: a gatekeeper for thrombus evaluation by delayed enhancement CMR. JACC Cardiovasc imaging. 2016; 9(5):505-515.Asinger RW, Mikell FL, Elsperger J, et al. incidence of leftventricular thrombosis after acute transmural myocardial infarction. Serial evaluation by two-dimensional echocardiography. N Engl J Med. 1981;305: 297-302.

14. Chiarella F, Santoro E, Domenicucci S, et al. Predischarge two-dimensional echocardiographic evaluation of left ventricular thrombosis after acute myocardial infarction in the GISSI-3 study. Am J Cardiol. 1998:81:822-7.

15. Solheim S, Seljeflot I, Lunde K, Bjornerheim R, Aakhus S, Forfang K, et al. Frequency of left ventricular Thrombus in patients with Anterior Wall acute myocardial infarction treated with percutaneous coronary intervention and dual antiplatelet therapy. Am J Cardiol. 2010;106(9):1197-200.

16. Mollet NR, Dymarkowski S, Volders W, Wathiong J, Herbots L, Rademakers $\mathrm{FE}$, et al. Visualization of ventricular thrombi with contrast-enhanced magnetic resonance imaging in patients with ischemic heart disease. Circulation. 2002;106(23):2873-6.

17. Weinsaft JW, Kim HW, Crowley AL, Klem I, Shenoy C, Van Assche L, et al. LV thrombus detection by routine echocardiography: insights into performance characteristics using delayed enhancement CMR. JACC Cardiovasc Imaging. 2011;4(7):702-12.

18. Tumkosit M, Martin CG, Bayram E, Morgan TM, Lane KS, Rerkpattanapipat P, et al. Left ventricular spherical remodeling and apical myocardial relaxation: cardiovascular MR imaging measurement of myocardial segments. Radiology. 2007;244(2):411-8.

Ready to submit your research? Choose BMC and benefit from:

- fast, convenient online submission

- thorough peer review by experienced researchers in your field

- rapid publication on acceptance

- support for research data, including large and complex data types

- gold Open Access which fosters wider collaboration and increased citations

- maximum visibility for your research: over $100 \mathrm{M}$ website views per year

At BMC, research is always in progress.

Learn more biomedcentral.com/submissions 